TAO, Vol. 16, No. 4, 709-729, October 2005

\title{
Geochemical Monitoring of Cold Waters during Seismicity: Implications for Earthquake-induced Modification in Shallow Aquifers
}

\author{
F. Italiano ${ }^{1, *}$, A. Caracausi $i^{1}$, R. Favara ${ }^{1}$, P. Innocenzi ${ }^{2}$ and G. Martinelli ${ }^{3}$
}

(Manuscript received 30 July 2004, in final form 29 July 2005)

\begin{abstract}
The geochemical monitoring carried out on fluids released in the Central Apennines (Umbria region) evidenced seismically-induced modifications of the physic-chemical parameters in all the released fluids, including some cold waters circulating in certain carbonate rocks that are exploited for drinking purposes. The results allowed us to recognize the presence of components of diverse origin that changed the chemical composition of the water. These components, potentially dangerous for human consumption, can be considered as being "secondary effects" on local aquifers, induced by crustal deformation.

We would also stress the relationship between the circulating fluids and active tectonic structures, as we detected modifications in some springs that were apparently not located anywhere near the local faults. The temporal variations in the geochemical features of the investigated cold waters are related to the local faulting activity that occurs during seismogenesis. The geochemical monitoring that provided information on fault movements during the seismic crisis, is also able to give us information regarding faulting activity in its earlier stages.
\end{abstract}

(Key words: Geochemical monitoring; Seismic events; Cold waters)

\section{INTRODUCTION}

This study has investigated the temporal variations that took place in the geochemistry of

${ }^{1}$ Istituto Nazionale di Geofisica e Vulcanologia, Sezione di Palermo, Palermo

${ }^{2}$ Valle Umbra Servizi - Foligno

${ }^{3}$ ARPA Regione Emilia-Romagna, Sezione di Reggio Emilia, Centro Eccellenza Acque, Reggio Emilia

* Corresponding author address: Dr. F. Italiano, Istituto Nazionale di Geofisica e Vulcanologia, Sezione di Palermo, Palermo; E-mail: f.italiano@pa.ingv.it 
some cold waters from the Umbria region (Central Apennines, Italy) with the aim of obtaining a better understanding of the relationship between seismogenic processes and the behaviour of circulating fluids.

On September 26, 1997, an earthquake struck the study-area (Fig. 1); this initiated a seismic crisis that lasted for more than six months. The seismic activity began with two main events of M 5.8 and 5.6 on 26 September, 1997, and was marked by five seismic shocks having a magnitude above 5 (Castello et al. 2005). Furthermore, hundreds of less violent events were recorded during the entire crisis (Amato et al. 1998, Morelli et al. 2000). Except for one event that occurred on March 26, 1998, (M 5.3; Castello et al. 2005), which was marked by a hypocenter located at the crust-mantle transition (as deep as $51 \mathrm{~km}$; Morelli et al. 2000), all the hypocenters of the entire seismic sequence were at a depth of $5-10 \mathrm{~km}$ in the upper crust, to which the crustal seismicity of the Umbria-Marche region is normally confined (Barchi 2002). The data on the active stress field indicate that this region is presently affected by crustal extension (La Vecchia et al. 1994; Montone et al. 1999), as the main seismogenic structures are a set of SW-dipping normal faults, antithetic to the main ENE-dipping normal fault of the central Apennines known as the Altotiberina Fault (Barchi et al. 1998).

A normal faulting marked the beginning of the seismic activity, while a compressive focal mechanism marked the $51 \mathrm{~km}$-deep 26 March, 1998 event (Morelli et al. 2000). The direct faulting activity allowed local permeability to increase and was responsible for the observed geochemical anomalies that were caused by an increased uprising of deep-originated fluids that affected the chemical and isotopic composition of the circulating fluids. Data regarding the variations of the ${ }^{3} \mathrm{He} /{ }^{4} \mathrm{He}$ ratio in gas vents, the chemical composition of some thermal waters and the modifications observed in the chemical and isotopic composition of the dissolved gases have already been published (Italiano et al. 2001; Favara et al. 2001; Italiano et al. 2004; Caracausi et al. 2005). Here, the results of the unexpected modifications in the chemical composition of some cold waters, exploited for drinking purposes, are presented. The observed modifications provide new information regarding the effects the development of a seismogenic process can have, and highlight the incidence of secondary-induced effects related to the seismicity, that may greatly increase the hazards that could be incurred by earthquake-prone areas.

\section{GEOLOGY AND HYDROGEOLOGY}

The Umbria region is a sector of the Northern-Apennines orogenic belt that was formed as a consequence of the collision between the European continental plate and the Adriatic-one (Barchi et al. 1998; Collettini and Barchi 2002). Geophysical and geological data highlight two main sectors within this area: the internal western belt (on the Tyrrhenian side), marked by active extensional tectonics, and the external one, marked by a coeval compressional stress field (Collettini and Barchi 2002). The investigated area is located between these two domains and represents a transitional zone due to the presence of an adjacent sector of compression and extension (Collettini and Barchi 2004). The stratigraphy of the area consists schematically of three main geo-lithological groups: (1) an Upper Paleozoic Phyllitic basement; (2) Upper Tri- 

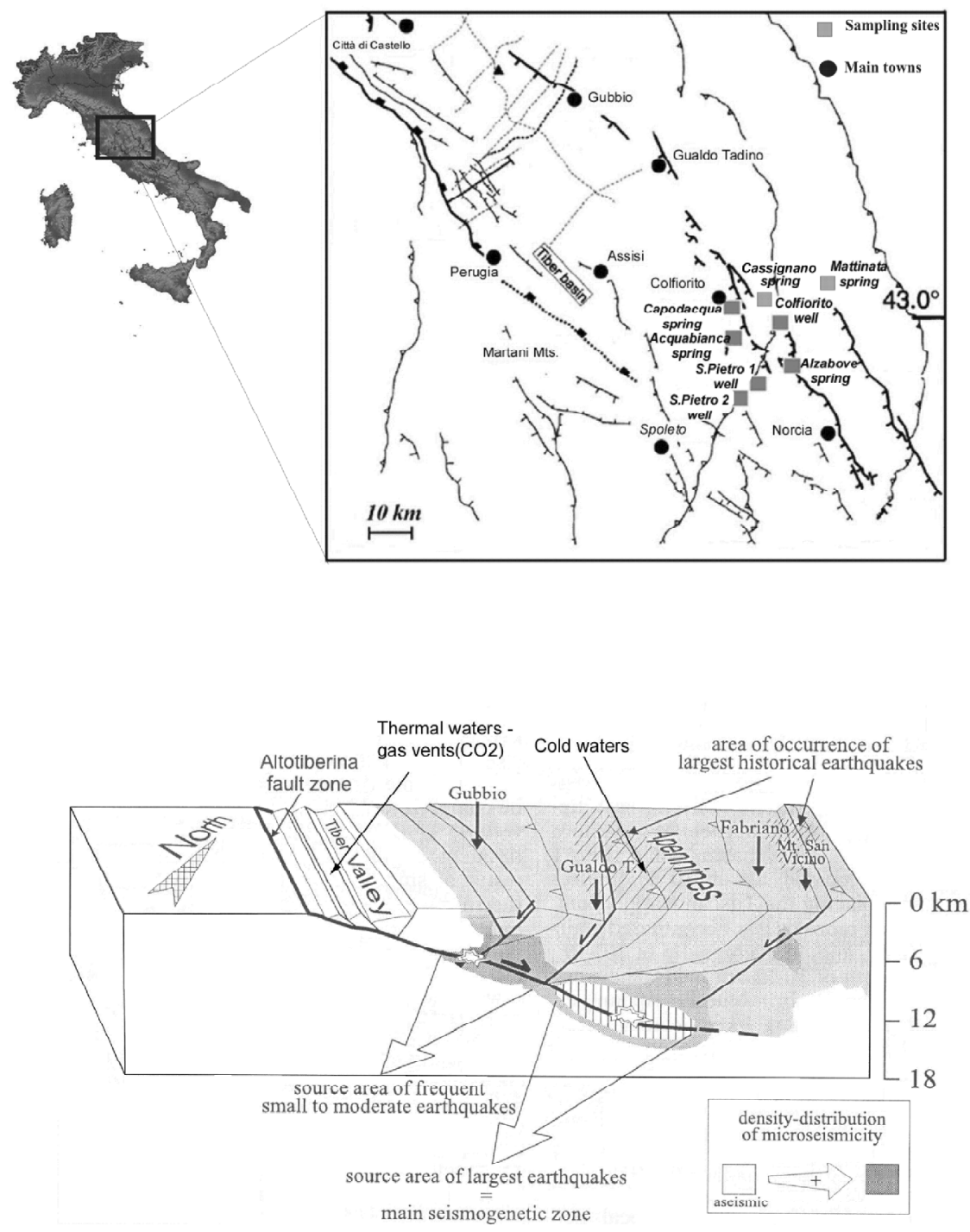

Fig. 1. Location of the sampling sites over the Umbria region (Central Apennines). The sketch map at the top-right side of the figure (modified after Collettini and Barchi, 2004) shows the relationships between the seismogenetic faults responsible for the last seismic crisis and the sampled cold waters. The 3D scheme at the bottom (modified after Boncio et al, 1998) displays the relative positioning of the main fault of the area (Altotiberina fault), the source area of the largest earthquakes and the extensive, seismogenetic, fault system. The sampling sites are located together with the occurrence of the thermal and mineral waters and the degassing areas. 
assic continental and shallow marine evaporitic sequences, a Lower Liassic carbonate platform and Jurassic-Paleogene pelagic cherty, marly and calcareous multilayers, which form a typical passive margin sequence; (3) a Lower Miocene-Pleistocene syn-tectonic turbiditic sequence (Barchi et al. 1998). Based on their mechanical properties, these groups have been subdivided into four "litho-structural units" whose sequence from top to bottom is Turbidites, Carbonate, Evaporite and Basement (Barchi et al. 1998).

From a hydrogeological point of view, it is possible to distinguish four main types of aquifers. The first is represented by the carbonate complexes of the "Carbonate litho-structural unit" that can be considered as being the best potential reservoir. Geomorphologic studies highlight that these complexes are characterized by a dense fault system and a widespread fracture-grid that supports infiltration of rainfall and circulation of water (Giaquinto et al. 1991; Zanzari et al. 2001). The second type is represented by alluvional deposits and the third by volcanic deposits. This latter is located in the Orvieto area. The fourth is a deep aquifer located in the evaporitic sequences (Quattrocchi et al. 2000; Zanzari et al. 2001).

\section{INVESTIGATED AREAS}

The sampled cold springs, i.e., Capodacqua, Alzabove, Acquabianca, Mattinate and Cassignano, are located in the carbonate complexes of the Foligno area (Fig. 1). Their flow rate ranges between 50 and $2501 \mathrm{~s} \mathrm{~s}^{-1}$. We also investigated two wells, S. Pietro 1 and S. Pietro 2, in the Foligno area and the well drilled in the Colfiorito high plain (Fig. 1). The S. Pietro 1 and S. Pietro 2 wells are about $190 \mathrm{~m}$ deep and cross alluvional deposits, while the Colfiorito well reaches a depth of $35 \mathrm{~m}$ where it strikes the aquifer located in the carbonate breccia (Bazzoffia et al. 2002).

The thermal springs are located in the carbonate complex. The Parrano thermal waters outflow at a rate of $0.011 \mathrm{~m}^{3} \mathrm{~s}^{-1}$. Both the Triponzo and Stifone hot springs are located along the Nera River valley, Triponzo at the bottom of the canyon generated by a system faults where the river itself flows, and Stifone in the southernmost end of the canyon. The latter discharges an amount of water estimated at about $10 \mathrm{~m}^{3} \mathrm{~s}^{-1}$ (Favara et al. 2001). The thermal waters are characterized by a constancy in flow rate and temperature (Stifone $=16.3^{\circ} \mathrm{C}$, Parrano $=26^{\circ} \mathrm{C}$ and Triponzo $=30^{\circ} \mathrm{C}$ ) that could be ascribed to feeding from large, deep reservoirs.

\section{SAMPLING AND ANALYTICAL METHODS}

The cold springs and wells were sampled at a frequency of once every three months between 1995 and 1997 and once every two months from the beginning of the 1997 seismic crisis until 2001. The cold water monitoring was related to quality control of drinking water and was carried out by the company "Valle Umbra Servizi".

Water temperature, $\mathrm{pH}$ and electrical conductivity were measured directly in the field. The temperature was always measured using the same mercury thermometer (scale $0-50^{\circ} \mathrm{C}$; resolution $0.1{ }^{\circ} \mathrm{C}$ ), while $\mathrm{pH}$ and electrical conductivity were measured by electronic instruments calibrated in situ using buffer solutions (error below $\pm 5 \%$ ). Water samples were filtered 
by cellulose filters $(0.45 \mu \mathrm{m})$ and the anions and cations measured in the laboratory by liquid cromatography (Dionex 2001) using a Dionex CS-12 and a Dionex AS4A-SC column for cation and anion determinations respectively. The $\mathrm{HCO}_{3}{ }^{-}$content was measured in the laboratory by standard titration procedures with $0.1 \mathrm{~N} \mathrm{HCl}$ on samples sealed in the field.

\section{RESULTS AND DISCUSSION}

The chemical features of most of the groundwaters in Umbria have already been assessed by other authors (e.g., Chiodini et al. 1982; Zanzari et al. 2001). The chemical composition of the sampled cold waters shows that it is heavily influenced by its circulation in carbonate rocks (main dissolved ions $=\mathrm{HCO}_{3}{ }^{-}$and $\mathrm{Ca}^{2+}$; Tables $1-8$ ). However, the composition of the thermal waters was considered consistent with a mixing of two water bodies (Chiodini et al. 1982): a shallower component (carbonate component) equilibrated with calcite and dolomite and a deeper one (selenite component) circulating in the Evaporitic Triassic basement. For a simple and quick classification of the sampled waters, the Langelier-Ludwig diagram (LL diagram; Fig. 2) was adopted (Langelier and Ludwig 1942) that displays data from both the cold and thermal waters collected before, during and after the 1997 - 98 seismic crisis.

The graph highlights the fact that all the waters underwent significant temporal variations regardless of their origin. No information regarding the temporal changes in the geochemical features was available until the occurrence of the last seismic crisis, when Favara et al. (2001) observed that the thermal waters underwent temporal modifications at the beginning of the main seismic sequence (from September 1997 to February 1998) and in coincidence with the 26 March, M 5.3 1998 event characterised by the deepest hypocentral depth. Unfortunately, no data regarding these waters before the onset of the crisis are available. In contrast, over the same period the chemical composition of the cold waters changed in coincidence with the extensional faulting activity that marked the beginning of the seismic sequence. The pre-seismic data allowed us to detect modifications that started some months before the first seismic shock. As previously pointed out (Heinicke et al. 2000; Italiano et al. 2001), during the seismic period enhanced $\mathrm{CO}_{2}$ degassing on a regional scale caused a drop in the $\mathrm{pH}$ of the thermal waters as well as changes in the mixing proportions between shallow and deep components (Favara et al. 2001).

In Fig. 2, an extended section of the lower part of the LL diagram is also shown. The analytical data highlight the seasonal variations that induced changes in the whole chemical composition (see the distribution of the plotted data) besides a wider variability due to an increased concentration of $\mathrm{HCO}_{3}{ }^{-}$shown by the horizontal distribution that was induced by phenomena not related to the local climatology. The observed variations follow similar trends for both cold and thermal waters (see also data on tables), leading us to conclude that a common origin, connected to the seismogenesis, accounts for the geochemical anomalies in all the groundwaters.

The ternary diagram $\mathrm{Cl}^{-}-\mathrm{SO}_{4}{ }^{2-}-\mathrm{HCO}_{3}{ }^{-}$of Fig. 3 shows that the chemical composition of all the waters (including thermal and cold waters), is determined by increasing concentrations of $\mathrm{SO}_{4}{ }^{2-}$ (for Triponzo thermal waters) and $\mathrm{Cl}^{-}$(for Parrano thermal waters.), or 
Table 1. Chemical composition of the Acquabianca spring water.

\begin{tabular}{|c|c|c|c|c|c|c|c|c|c|c|c|}
\hline Date & $T^{\circ} \mathrm{C}$ & $p H$ & Cond & $\mathrm{HCO}_{3}^{-}$ & $C l$ & $\mathrm{SO}_{4}{ }^{2-}$ & $\mathrm{Ca}^{2+}$ & $M g^{2+}$ & $\boldsymbol{K}^{+}$ & $\mathrm{Na}^{+}$ & $\mathrm{Fe}$ \\
\hline $10 / 02 / 1993$ & n.a & 7.41 & 368 & n.a & 0.20 & 0.07 & 4.3 & 0.30 & $1.79 \mathrm{E}-02$ & 0.17 & n.a \\
\hline $27 / 09 / 1993$ & n.a & 7.37 & 409 & n.a & 0.15 & 0.06 & 4.0 & 0.20 & $1.79 \mathrm{E}-02$ & 0.18 & n.a \\
\hline $06 / 02 / 1995$ & n.a & 7.41 & 364 & 3.5 & 0.24 & 0.08 & 3.2 & 0.22 & $2.31 \mathrm{E}-02$ & 0.15 & n.a \\
\hline $10 / 05 / 1995$ & 11.7 & 7.68 & 489 & 3.5 & 0.20 & 0.06 & 4.2 & 0.58 & $1.79 \mathrm{E}-02$ & 0.16 & 2.3 \\
\hline $14 / 12 / 1995$ & 11.4 & 7.58 & 491 & 3.8 & 0.20 & 0.06 & 4.6 & 0.09 & $1.03 \mathrm{E}-02$ & 0.15 & 2.3 \\
\hline $17 / 06 / 1996$ & 12.0 & 7.72 & 478 & 3.7 & 0.17 & 0.07 & 4.5 & 0.10 & $1.54 \mathrm{E}-02$ & 0.14 & 1.1 \\
\hline $15 / 10 / 1996$ & 11.8 & 7.68 & 478 & 3.5 & 0.19 & 0.07 & 4.4 & 0.11 & $2.20 \mathrm{E}-02$ & 0.14 & 1.2 \\
\hline $12 / 06 / 1997$ & 12.1 & 7.74 & 482 & 3.8 & 0.19 & 0.05 & 4.2 & 0.14 & $1.77 \mathrm{E}-02$ & 0.16 & 4.9 \\
\hline $27 / 09 / 1997$ & 12.2 & 7.31 & 507 & 3.8 & 0.17 & 0.16 & 4.4 & 0.16 & $1.82 \mathrm{E}-02$ & 0.16 & 5.6 \\
\hline $04 / 10 / 1997$ & 11.7 & 7.11 & 555 & 3.8 & 0.19 & 0.06 & 4.5 & 0.22 & $2.26 \mathrm{E}-02$ & 0.18 & 6.2 \\
\hline $14 / 10 / 1997$ & 11.6 & 7.23 & 548 & 3.8 & 0.18 & 0.05 & 4.5 & 0.21 & $2.18 \mathrm{E}-02$ & 0.17 & 6.1 \\
\hline $22 / 10 / 1997$ & 11.7 & 7.28 & 545 & 3.5 & 0.18 & 0.05 & 4.5 & 0.23 & $1.97 \mathrm{E}-02$ & 0.17 & 6.0 \\
\hline $18 / 11 / 1997$ & 11.9 & 7.76 & 495 & 3.6 & 0.19 & 0.05 & 4.2 & 0.14 & $1.74 \mathrm{E}-02$ & 0.16 & 4.9 \\
\hline $23 / 03 / 1998$ & 11.2 & 7.49 & 418 & 3.5 & 0.17 & 0.07 & 4.2 & 0.16 & $1.69 \mathrm{E}-02$ & 0.17 & 4.6 \\
\hline $13 / 05 / 1998$ & 12.0 & 7.51 & 423 & 3.5 & 0.17 & 0.07 & 4.1 & 0.15 & $1.67 \mathrm{E}-02$ & 0.16 & 4.7 \\
\hline $18 / 05 / 1998$ & 11.8 & 7.67 & 453 & 3.6 & 0.13 & 0.06 & 4.1 & 0.14 & $2.13 \mathrm{E}-02$ & 0.16 & 4.6 \\
\hline $15 / 12 / 1998$ & 11.3 & 7.40 & 425 & 3.5 & 0.19 & 0.06 & 4.2 & 0.16 & $1.79 \mathrm{E}-02$ & 0.17 & 3.2 \\
\hline $29 / 03 / 1999$ & 11.7 & 7.52 & 425 & 3.5 & 0.18 & 0.07 & 4.2 & 0.14 & $1.82 \mathrm{E}-02$ & 0.16 & 3.2 \\
\hline $24 / 06 / 1999$ & 12.2 & 7.33 & 380 & 3.5 & 0.16 & 0.05 & 4.2 & 0.15 & $2.05 \mathrm{E}-02$ & 0.16 & 3.5 \\
\hline $28 / 09 / 1999$ & 12.1 & 7.48 & 405 & 3.6 & 0.17 & 0.06 & 4.2 & 0.13 & $2.00 \mathrm{E}-02$ & 0.17 & 2.9 \\
\hline $11 / 12 / 1999$ & 11.6 & 7.38 & 413 & 3.6 & 0.17 & 0.06 & 4.2 & 0.14 & $1.79 \mathrm{E}-02$ & 0.17 & 3.1 \\
\hline $24 / 03 / 2000$ & 11.7 & 7.46 & 350 & 3.5 & 0.15 & 0.05 & 4.3 & 0.15 & $1.90 \mathrm{E}-02$ & 0.16 & 2.1 \\
\hline $24 / 06 / 2000$ & 11.9 & 7.42 & 396 & 3.5 & 0.17 & 0.07 & 4.1 & 0.13 & $2.31 \mathrm{E}-02$ & 0.14 & 2.9 \\
\hline $25 / 09 / 2000$ & 12.4 & 7.48 & 390 & 3.7 & 0.16 & 0.06 & 4.1 & 0.13 & $2.26 \mathrm{E}-02$ & 0.17 & 3.0 \\
\hline $09 / 12 / 2000$ & 11.1 & 7.48 & 388 & 3.6 & 0.16 & 0.06 & 4.3 & 0.15 & $2.31 \mathrm{E}-02$ & 0.21 & 3.1 \\
\hline $24 / 03 / 2001$ & 12.0 & 7.51 & 424 & 3.5 & 0.18 & 0.08 & 4.2 & 0.14 & $2.13 \mathrm{E}-02$ & 0.17 & 3.3 \\
\hline $21 / 05 / 2001$ & 11.8 & 7.52 & 421 & 3.6 & 0.19 & 0.08 & 4.1 & 0.14 & $2.05 \mathrm{E}-02$ & 0.14 & 3.4 \\
\hline $06 / 09 / 2001$ & 12.9 & 7.49 & 418 & 3.6 & 0.17 & 0.07 & 4.2 & 0.15 & $2.33 \mathrm{E}-02$ & 0.14 & 3.2 \\
\hline $18 / 02 / 2002$ & 11.0 & 7.40 & 376 & 3.6 & 0.17 & 0.09 & 4.6 & 0.15 & $1.79 \mathrm{E}-02$ & 0.18 & 4.3 \\
\hline $28 / 06 / 2002$ & 13.0 & 7.55 & 374 & 3.5 & 0.15 & 0.04 & 4.4 & 0.15 & $1.28 \mathrm{E}-02$ & 0.13 & 2.4 \\
\hline $28 / 12 / 2002$ & 11.8 & 7.50 & 401 & 3.5 & 0.17 & 0.06 & 4.4 & 0.17 & $2.31 \mathrm{E}-02$ & 0.19 & 2.5 \\
\hline
\end{tabular}

note: Cond $=$ electrical conductivity in $\mu \mathrm{S} \mathrm{cm}^{-1}$; ions and cations concentration in meq $1^{-1}$; iron in $\mu \mathrm{g}^{-1}$; n.a. $=$ not analysed. 
Table 2. Chemical composition of the Capodacqua spring water.

\begin{tabular}{|c|c|c|c|c|c|c|c|c|c|c|c|}
\hline date & $T^{\circ} \mathrm{C}$ & $p H$ & Cond & $\mathrm{HCO}_{3}^{-}$ & $C r$ & $\mathrm{SO}_{4}^{2-}$ & $\mathrm{Ca}^{2+}$ & $\mathrm{Mg}^{2+}$ & $\boldsymbol{K}^{+}$ & $\mathrm{Na}^{+}$ & $\mathrm{Fe}$ \\
\hline $10 / 05 / 1995$ & 11.8 & 7.55 & 480 & 3.34 & 0.17 & 0.08 & 4.07 & 0.16 & $1.54 \mathrm{E}-02$ & 0.16 & 2.3 \\
\hline $14 / 12 / 1995$ & 10.7 & 7.45 & 463 & 3.44 & 0.19 & 0.09 & 4.25 & 0.15 & $1.26 \mathrm{E}-02$ & 0.16 & 2.3 \\
\hline $17 / 06 / 1996$ & 12.1 & 7.80 & 455 & 3.36 & 0.19 & 0.09 & 4.15 & 0.16 & $1.79 \mathrm{E}-02$ & 0.13 & 1.0 \\
\hline $15 / 10 / 1996$ & 11.6 & 7.67 & 464 & 3.34 & 0.19 & 0.09 & 4.10 & 0.16 & $2.23 \mathrm{E}-02$ & 0.11 & 1.9 \\
\hline $12 / 06 / 1997$ & 11.8 & 7.65 & 496 & 3.31 & 0.18 & 0.08 & 4.06 & 0.17 & $1.56 \mathrm{E}-02$ & 0.16 & 4.8 \\
\hline $27 / 09 / 1997$ & 12.1 & 7.53 & 490 & 3.36 & 0.19 & 0.15 & 4.06 & 0.17 & $1.51 \mathrm{E}-02$ & 0.15 & 5.0 \\
\hline $05 / 10 / 1997$ & 12.3 & 7.49 & 551 & 3.54 & 0.21 & 0.11 & 4.32 & 0.18 & $1.54 \mathrm{E}-02$ & 0.16 & 5.5 \\
\hline $14 / 10 / 1997$ & 17.5 & 7.54 & 553 & 3.57 & 0.21 & 0.10 & 4.37 & 0.20 & $1.59 \mathrm{E}-02$ & 0.16 & 5.4 \\
\hline $18 / 11 / 1997$ & 11.3 & 7.58 & 553 & 3.57 & 0.19 & 0.09 & 4.36 & 0.19 & $1.61 \mathrm{E}-02$ & 0.16 & 4.8 \\
\hline $23 / 03 / 1998$ & 11.6 & 7.60 & 398 & 3.31 & 0.14 & 0.08 & 4.06 & 0.16 & $1.54 \mathrm{E}-02$ & 0.16 & 4.5 \\
\hline $13 / 05 / 1998$ & 12.0 & 7.62 & 397 & 3.28 & 0.13 & 0.08 & 4.02 & 0.15 & $1.67 \mathrm{E}-02$ & 0.15 & 4.5 \\
\hline $18 / 05 / 1998$ & 11.6 & 7.64 & 373 & 3.28 & 0.13 & 0.08 & 4.02 & 0.15 & $1.74 \mathrm{E}-02$ & 0.15 & 4.6 \\
\hline $06 / 06 / 1998$ & 11.7 & 7.52 & 333 & 3.25 & 0.15 & 0.09 & 4.00 & 0.15 & $1.59 \mathrm{E}-02$ & 0.16 & 4.0 \\
\hline $15 / 12 / 1998$ & 10.7 & 7.57 & 396 & 3.31 & 0.18 & 0.08 & 4.05 & 0.17 & $1.54 \mathrm{E}-02$ & 0.16 & 2.8 \\
\hline $29 / 03 / 1999$ & 12.6 & 7.74 & 398 & 3.31 & 0.17 & 0.08 & 4.05 & 0.16 & $1.69 \mathrm{E}-02$ & 0.15 & 3.0 \\
\hline $24 / 06 / 1999$ & 12.2 & 7.45 & 406 & 3.33 & 0.17 & 0.11 & 4.06 & 0.18 & $1.79 \mathrm{E}-02$ & 0.13 & 3.1 \\
\hline $28 / 09 / 1999$ & 12.2 & 7.43 & 387 & 3.31 & 0.17 & 0.08 & 4.05 & 0.16 & $1.74 \mathrm{E}-02$ & 0.17 & 2.9 \\
\hline $11 / 12 / 1999$ & 10.8 & 7.60 & 399 & 3.36 & 0.17 & 0.09 & 4.10 & 0.17 & $1.54 \mathrm{E}-02$ & 0.33 & 3.0 \\
\hline $24 / 03 / 2000$ & 11.5 & 7.47 & 380 & 3.33 & 0.16 & 0.08 & 4.06 & 0.17 & $1.85 \mathrm{E}-02$ & 0.13 & 2.6 \\
\hline $24 / 06 / 2000$ & 13.7 & 7.46 & 394 & 3.31 & 0.17 & 0.11 & 4.05 & 0.19 & $2.31 \mathrm{E}-02$ & 0.12 & 2.7 \\
\hline $25 / 09 / 2000$ & 11.7 & 7.37 & 368 & 3.33 & 0.16 & 0.08 & 4.06 & 0.16 & $1.82 \mathrm{E}-02$ & 0.19 & 2.3 \\
\hline $09 / 12 / 2000$ & 10.4 & 7.51 & 398 & 3.33 & 0.20 & 0.10 & 4.07 & 0.18 & $1.79 \mathrm{E}-02$ & 0.23 & 2.4 \\
\hline $24 / 03 / 2001$ & 11.7 & 7.48 & 408 & 3.31 & 0.17 & 0.10 & 4.05 & 0.17 & $1.90 \mathrm{E}-02$ & 0.15 & 2.4 \\
\hline $25 / 05 / 2001$ & 11.8 & 7.47 & 404 & 3.34 & 0.17 & 0.10 & 4.08 & 0.18 & $2.05 \mathrm{E}-02$ & 0.13 & 2.5 \\
\hline $12 / 09 / 2001$ & 12.8 & 7.48 & 406 & 3.33 & 0.16 & 0.10 & 4.07 & 0.16 & $2.00 \mathrm{E}-02$ & 0.15 & 2.4 \\
\hline $27 / 12 / 2001$ & 14.3 & 7.69 & 357 & 3.38 & 0.14 & 0.09 & 4.11 & 0.18 & $1.79 \mathrm{E}-02$ & 0.16 & 3.0 \\
\hline
\end{tabular}

note: Cond $=$ electrical conductivity in $\mu \mathrm{S} \mathrm{cm}^{-1}$; ions and cations concentration in meq $1^{-1}$; iron in $\mu \mathrm{g} 1^{-1}$. 
Table 3. Chemical composition of the Alzabove spring water.

\begin{tabular}{|c|c|c|c|c|c|c|c|c|c|c|c|}
\hline Date & $T^{\circ} \mathrm{C}$ & $p H$ & Cond & $\mathrm{HCO}_{3}^{-}$ & $\mathrm{Cl}$ & $\mathrm{SO}_{4}{ }^{2-}$ & $C a^{2+}$ & $\mathrm{Mg}^{2+}$ & $\boldsymbol{K}^{+}$ & $\mathrm{Na}^{+}$ & $\mathrm{Fe}$ \\
\hline $16 / 10 / 1992$ & n.a & 7.32 & 387 & n.a & 0.11 & 0.06 & 3.99 & 0.20 & $2.05 \mathrm{E}-02$ & 0.14 & n.a \\
\hline $02 / 05 / 1993$ & n.a & 7.39 & 387 & n.a & 0.10 & 0.06 & 3.99 & 0.20 & $2.05 \mathrm{E}-02$ & 0.21 & n.a \\
\hline $21 / 09 / 1993$ & n.a & 7.41 & 411 & n.a & 0.10 & 0.14 & 3.99 & 0.10 & $1.54 \mathrm{E}-02$ & 0.18 & n.a \\
\hline $13 / 02 / 1995$ & п.a & 7.63 & 383 & n.a & 0.14 & 0.12 & 4.07 & 0.08 & $2.05 \mathrm{E}-02$ & 0.26 & n.a \\
\hline $10 / 05 / 1995$ & 10.6 & 7.46 & 473 & 3.67 & 0.16 & 0.11 & 4.17 & 0.18 & $1.54 \mathrm{E}-02$ & 0.14 & 3.8 \\
\hline $14 / 12 / 1995$ & 10.1 & 7.36 & 471 & 3.64 & 0.16 & 0.11 & 4.14 & 0.17 & $1.03 \mathrm{E}-02$ & 0.14 & 2.8 \\
\hline $17 / 06 / 1996$ & 12.0 & 7.49 & 471 & 3.85 & 0.16 & 0.11 & 4.42 & 0.17 & $1.79 \mathrm{E}-02$ & 0.13 & 1.1 \\
\hline $15 / 10 / 1996$ & 11.2 & 7.44 & 470 & 3.77 & 0.16 & 0.11 & 4.38 & 0.17 & $2.54 \mathrm{E}-02$ & 0.13 & 1.2 \\
\hline $12 / 06 / 1997$ & 11.9 & 7.63 & 473 & 3.64 & 0.15 & 0.11 & 4.18 & 0.18 & $1.56 \mathrm{E}-02$ & 0.14 & 5.5 \\
\hline $27 / 09 / 1997$ & 12.0 & 7.58 & 532 & 3.87 & 0.18 & 0.45 & 4.40 & 0.20 & $2.82 \mathrm{E}-02$ & 0.14 & 6.5 \\
\hline 03/10/1997 & 12.2 & 7.23 & 447 & 3.82 & 0.17 & 0.11 & 4.37 & 0.19 & $2.56 \mathrm{E}-02$ & 0.13 & 5.6 \\
\hline $14 / 10 / 1997$ & 13.1 & 7.37 & 537 & 3.85 & 0.16 & 0.11 & 4.38 & 0.18 & $2.31 \mathrm{E}-02$ & 0.14 & 5.5 \\
\hline $18 / 11 / 1997$ & 11.2 & 7.63 & 535 & 3.74 & 0.17 & 0.12 & 4.19 & 0.20 & $1.61 \mathrm{E}-02$ & 0.14 & 5.5 \\
\hline 23/03/1998 & 10.2 & 7.65 & 410 & 3.77 & 0.14 & 0.10 & 4.27 & 0.17 & $1.41 \mathrm{E}-02$ & 0.15 & 3.3 \\
\hline $13 / 05 / 1998$ & 10.8 & 7.66 & 411 & 3.74 & 0.14 & 0.10 & 4.26 & 0.18 & $1.67 \mathrm{E}-02$ & 0.13 & 3.2 \\
\hline $18 / 05 / 1998$ & 10.2 & 7.64 & 413 & 3.64 & 0.15 & 0.11 & 4.15 & 0.17 & $1.67 \mathrm{E}-02$ & 0.14 & 3.4 \\
\hline $15 / 12 / 1998$ & 10.2 & 7.49 & 412 & 3.69 & 0.16 & 0.06 & 4.20 & 0.21 & $1.54 \mathrm{E}-02$ & 0.15 & 2.4 \\
\hline 29/03/1999 & 7.5 & 7.57 & 423 & 3,44 & 0.16 & 0.06 & 3.94 & 0.21 & $2.82 \mathrm{E}-02$ & 0.14 & 3.0 \\
\hline $24 / 06 / 1999$ & 12.8 & 7.69 & 373 & 3.33 & 0.16 & 0.07 & 3.82 & 0.12 & $2.31 \mathrm{E}-02$ & 0.16 & 2.9 \\
\hline 28/09/1999 & 10.9 & 7.36 & 407 & 3.61 & 0.15 & 0.12 & 4.10 & 0.20 & $2.82 \mathrm{E}-02$ & 0.14 & 2.6 \\
\hline $11 / 12 / 1999$ & 10.8 & 7.55 & 404 & 3.77 & 0.15 & 0.13 & 4.33 & 0.17 & $2.82 \mathrm{E}-02$ & 0.31 & 2.0 \\
\hline $24 / 03 / 2000$ & 10.8 & 7.39 & 388 & 3.74 & 0.15 & 0.09 & 4.27 & 0.18 & $2.31 \mathrm{E}-02$ & 0.15 & 3.0 \\
\hline $24 / 06 / 2000$ & 10.5 & 7.66 & 395 & 3.34 & 0.15 & 0.09 & 3.79 & 0.14 & $2.82 \mathrm{E}-02$ & 0.15 & 5.1 \\
\hline $12 / 08 / 2000$ & 10.9 & 7.35 & 406 & 3.59 & 0.16 & 0.12 & 4.10 & 0.15 & $3.08 \mathrm{E}-02$ & 0.15 & 3.4 \\
\hline $26 / 09 / 2000$ & 10.7 & 7.62 & 385 & 3.61 & 0.15 & 0.12 & 4.11 & 0.16 & $1.54 \mathrm{E}-02$ & 0.18 & 3.0 \\
\hline $09 / 12 / 2000$ & 9.8 & 7.68 & 396 & 3.75 & 0.16 & 0.13 & 4.27 & 0.18 & $1.28 \mathrm{E}-02$ & 0.23 & 3.4 \\
\hline $24 / 03 / 2001$ & 10.6 & 7.65 & 418 & 3.59 & 0.16 & 0.11 & 4.09 & 0.14 & $2.05 \mathrm{E}-02$ & 0.14 & 4.3 \\
\hline $21 / 05 / 2001$ & 10.7 & 7.64 & 416 & 3.34 & 0.16 & 0.09 & 3.81 & 0.12 & $3.85 \mathrm{E}-02$ & 0.15 & 4.6 \\
\hline $12 / 09 / 2001$ & 11.5 & 7.54 & 423 & 3.51 & 0.16 & 0.10 & 3.98 & 0.17 & $3.08 \mathrm{E}-02$ & 0.15 & 3.2 \\
\hline $29 / 12 / 2001$ & 10.8 & 7.47 & 367 & 3.69 & 0.10 & 0.13 & 4.19 & 0.24 & $3.00 \mathrm{E}-02$ & 0.17 & 3.1 \\
\hline $19 / 02 / 2002$ & 10.8 & 7.47 & 367 & n.a & n.a & n.a & n.a & n.a & n.a & n.a & 3.1 \\
\hline $28 / 06 / 2002$ & 11.1 & 7.72 & 365 & n.a & n.a & n.a & n.a & n.a & n.a & n. a & 4.8 \\
\hline $28 / 12 / 2002$ & 10.0 & 7.42 & 390 & n.a & n.a & n.a & n.a & n.a & n.a & n.a & 2.4 \\
\hline
\end{tabular}

note: Cond $=$ electrical conductivity in $\mu \mathrm{S} \mathrm{cm}^{-1}$; ions and cations concentration in meq $1^{-1}$; iron in $\mu \mathrm{g}^{-1}$; n.a. $=$ not analysed. 
Table 4. Chemical composition of the Colfiorito well water.

\begin{tabular}{|c|c|c|c|c|c|c|c|c|c|c|c|}
\hline Date & $T^{\circ} \mathrm{C}$ & $p H$ & Cond & $\mathrm{HCO}_{3}^{-}$ & $C t$ & $\mathrm{SO}_{4}^{2-}$ & $\mathrm{Ca}^{2+}$ & $\mathrm{Mg}^{2+}$ & $\boldsymbol{K}^{+}$ & $\mathrm{Na}^{+}$ & $F e$ \\
\hline $10 / 05 / 1995$ & 11.4 & 7.62 & 453 & 3.07 & 0.19 & 0.07 & 3.84 & 0.11 & 0.02 & 0.17 & 4.4 \\
\hline $14 / 12 / 1995$ & 10.1 & 7.41 & 441 & 3.11 & 0.17 & 0.07 & 3.89 & 0.11 & 0.02 & 0.18 & 3.2 \\
\hline $17 / 06 / 1996$ & 11.9 & 7.70 & 431 & 3.13 & 0.16 & 0.06 & 3.91 & 0.10 & 0.02 & 0.18 & 2.0 \\
\hline $15 / 10 / 1996$ & 11.2 & 7.56 & 431 & 3.16 & 0.17 & 0.06 & 3.96 & 0.09 & 0.03 & 0.19 & 2.2 \\
\hline $12 / 06 / 1997$ & 11.8 & 7.70 & 464 & 3.05 & 0.19 & 0.06 & 3.81 & 0.11 & 0.02 & 0.17 & 5.3 \\
\hline $27 / 09 / 1997$ & 11.8 & 7.16 & 698 & 3.41 & 0.15 & 0.18 & 4.27 & 0.11 & 0.03 & 0.20 & 6.0 \\
\hline $30 / 09 / 1997$ & 11.6 & 7.45 & 513 & 3.25 & 0.22 & 0.07 & 4.07 & 0.11 & 0.03 & 0.19 & 5.0 \\
\hline $18 / 11 / 1997$ & 11.0 & 7.72 & 489 & 3.11 & 0.19 & 0.07 & 3.83 & 0.11 & 0.02 & 0.17 & 5.3 \\
\hline $23 / 03 / 1998$ & 11.0 & 7.63 & 375 & 3.10 & 0.16 & 0.06 & 3.83 & 0.10 & 0.02 & 0.16 & 3.0 \\
\hline $13 / 05 / 1998$ & 11.3 & 7.62 & 371 & 3.11 & 0.16 & 0.07 & 3.84 & 0.11 & 0.02 & 0.16 & 3.0 \\
\hline $18 / 05 / 1998$ & 11.6 & 7.60 & 385 & 2.95 & 0.14 & 0.05 & 3.68 & 0.10 & 0.03 & 0.17 & 2.5 \\
\hline $15 / 12 / 1998$ & 10.4 & 7.55 & 370 & 3.07 & 0.18 & 0.05 & 3.83 & 0.12 & 0.03 & 0.17 & 2.2 \\
\hline 29/03/1999 & 8.6 & 7.74 & 340 & 3.10 & 0.14 & 0.06 & 3.83 & 0.12 & 0.02 & 0.17 & 2.5 \\
\hline $24 / 06 / 1999$ & 12.8 & 7.69 & 373 & 3.07 & 0.16 & 0.07 & 3.82 & 0.12 & 0.03 & 0.17 & 2.6 \\
\hline 28/09/1999 & 11.7 & 7.45 & 370 & 3.05 & 0.16 & 0.06 & 3.82 & 0.12 & 0.03 & 0.17 & 2.7 \\
\hline $11 / 12 / 1999$ & 10.4 & 7.69 & 380 & 3.08 & 0.16 & 0.05 & 3.82 & 0.13 & 0.02 & 0.35 & 2.1 \\
\hline $24 / 03 / 2000$ & 11.5 & 7.52 & 354 & 3.05 & 0.15 & 0.06 & 3.82 & 0.12 & 0.03 & 0.21 & 2.5 \\
\hline $24 / 06 / 2000$ & 12.0 & 7.68 & 356 & 3.00 & 0.14 & 0.07 & 3.75 & 0.14 & 0.03 & 0.17 & 1.9 \\
\hline $25 / 09 / 2000$ & 11.5 & 7.64 & 348 & 3.05 & 0.15 & 0.06 & 3.83 & 0.13 & 0.02 & 0.20 & 2.0 \\
\hline $09 / 12 / 2000$ & 10.2 & 7.75 & 358 & 3.00 & 0.17 & 0.05 & 3.73 & 0.12 & 0.01 & 0.24 & 2.2 \\
\hline $24 / 03 / 2001$ & 11.0 & 7.67 & 385 & 3.05 & 0.17 & 0.06 & 3.82 & 0.11 & 0.03 & 0.18 & 2.0 \\
\hline $21 / 05 / 2001$ & 11.0 & 7.65 & 380 & 2.95 & 0.17 & 0.07 & 3.70 & 0.13 & 0.02 & 0.15 & 1.8 \\
\hline $12 / 09 / 2001$ & 12.0 & 7.55 & 376 & 2.98 & 0.16 & 0.06 & 3.73 & 0.19 & 0.03 & 0.16 & 2.0 \\
\hline $29 / 12 / 2001$ & 10.4 & 7.54 & 277 & 3.54 & 0.16 & 0.05 & 3.95 & 0.09 & 0.02 & 0.17 & 2.2 \\
\hline $18 / 02 / 2002$ & n.a & n.a & n.a & n.a & n.a & n.a & n.a & n.a & n.a & n.a & 2.2 \\
\hline $28 / 06 / 2002$ & n.a & n.a & n.a & n.a & n.a & n.a & n.a & n.a & n.a & n.a & 1.2 \\
\hline $28 / 12 / 2002$ & n.a & n.a & n.a & n.a & n.a & n.a & n.a & n.a & n.a & n.a & 4.4 \\
\hline
\end{tabular}

note: Cond $=$ electrical conductivity in $\mu \mathrm{S} \mathrm{cm}^{-1}$; ions and cations concentration in meq $\mathrm{l}^{-1}$; iron in $\mu \mathrm{g}^{-1}$. 
Table 5. Chemical composition of the S. Pietro 1 well water.

\begin{tabular}{|c|c|c|c|c|c|c|c|c|c|c|c|}
\hline Date & $T^{\circ} \mathrm{C}$ & $p H$ & Cond & $\mathrm{HCO}_{3}^{-}$ & $C t$ & $\mathrm{SO}_{4}^{2-}$ & $\mathrm{Ca}^{2+}$ & $\mathbf{M g}^{2+}$ & $K^{+}$ & $\mathrm{Na}^{+}$ & $F e$ \\
\hline $10 / 05 / 1995$ & 13.5 & 7.36 & 660 & 3.85 & 0.31 & 1.17 & 4.97 & 0.51 & $1.05 \mathrm{E}-01$ & 0.44 & 6.2 \\
\hline $14 / 12 / 1995$ & 11.8 & 7.30 & 662 & 4.30 & 0.31 & 1.13 & 5.61 & 0.43 & $1.72 \mathrm{E}-01$ & 0.53 & 6.2 \\
\hline $17 / 06 / 1996$ & 13.6 & 7.71 & 655 & 4.26 & 0.32 & 1.11 & 5.50 & 0.69 & $6.66 \mathrm{E}-02$ & 0.49 & 4.2 \\
\hline $15 / 10 / 1996$ & 13.3 & 7.64 & 659 & 3.89 & 0.33 & 1.12 & 5.01 & 0.84 & $4.61 \mathrm{E}-02$ & 0.47 & 5.7 \\
\hline $12 / 06 / 1997$ & 13.9 & 7.68 & 656 & 3.82 & 0.31 & 1.08 & 4.91 & 0.76 & $4.26 \mathrm{E}-02$ & 0.46 & 15.4 \\
\hline $27 / 09 / 1997$ & 13.7 & 7.38 & 694 & 4.10 & 0.53 & 2.10 & 5.29 & 0.87 & $4.77 \mathrm{E}-02$ & 0.54 & 24.0 \\
\hline $05 / 10 / 1997$ & 14.0 & 7.30 & 755 & 4.34 & 0.37 & 1.13 & 5.59 & 0.95 & $5.00 \mathrm{E}-02$ & 0.49 & 18.0 \\
\hline $18 / 11 / 1997$ & 14.0 & 7.78 & 668 & 4.05 & 0.34 & 1.13 & 5.19 & 0.85 & $4.51 \mathrm{E}-02$ & 0.47 & 16.5 \\
\hline 23/03/1998 & 13.3 & 7.32 & 580 & 3.89 & 0.27 & 1.08 & 4.93 & 0.75 & $4.36 \mathrm{E}-02$ & 0.47 & 13.4 \\
\hline $13 / 05 / 1998$ & 13.6 & 7.34 & 575 & 3.85 & 0.27 & 1.08 & 4.95 & 0.74 & $4.46 \mathrm{E}-02$ & 0.47 & 10.0 \\
\hline $18 / 05 / 1998$ & 13.2 & 7.68 & 569 & 3.84 & 0.27 & 1.08 & 4.92 & 0.76 & $4.36 \mathrm{E}-02$ & 0.47 & 8.8 \\
\hline $15 / 12 / 1998$ & 9.5 & 7.42 & 570 & 4.16 & 0.29 & 0.97 & 5.34 & 0.74 & 4.61E-02 & 0.48 & 6.5 \\
\hline $29 / 03 / 1999$ & 13.6 & 7.46 & 577 & 4.03 & 0.27 & 1.01 & 5.19 & 0.72 & $4.49 \mathrm{E}-02$ & 0.48 & 6.2 \\
\hline $24 / 06 / 1999$ & 13.9 & 7.26 & 544 & 4.10 & 0.35 & 1.14 & 5.29 & 0.73 & $4.10 \mathrm{E}-02$ & 0.49 & 6.6 \\
\hline 28/09/1999 & 13.7 & 7.28 & 554 & 4.07 & 0.35 & 1.09 & 5.24 & 0.72 & $4.23 \mathrm{E}-02$ & 0.48 & 5.2 \\
\hline $11 / 12 / 1999$ & 13.1 & 7.41 & 560 & 3.95 & 0.38 & 1.11 & 5.09 & 0.71 & $4.10 \mathrm{E}-02$ & 0.61 & 5.9 \\
\hline $24 / 03 / 2000$ & 13.1 & 7.32 & 510 & 4.07 & 0.27 & 0.97 & 5.24 & 0.72 & $4.36 \mathrm{E}-02$ & 0.56 & 4.8 \\
\hline $24 / 06 / 2000$ & 14.9 & 7.46 & 534 & 3.87 & 0.30 & 1.01 & 4.98 & 0.70 & $4.61 \mathrm{E}-02$ & 0.53 & 6.9 \\
\hline $25 / 09 / 2000$ & 13.8 & 7.54 & 528 & 3.98 & 0.28 & 0.98 & 5.14 & 0.71 & $4.61 \mathrm{E}-02$ & 0.52 & 5.0 \\
\hline $09 / 12 / 2000$ & 12.0 & 8.08 & 457 & 4.00 & 0.33 & 1.03 & 5.14 & 0.72 & $5.38 \mathrm{E}-02$ & 0.53 & 6.7 \\
\hline $24 / 03 / 2001$ & 13.7 & 7.56 & 568 & 4.03 & 0.33 & 1.04 & 5.19 & 0.71 & $5.64 \mathrm{E}-02$ & 0.50 & 6.8 \\
\hline $21 / 05 / 2001$ & 14.3 & 7.54 & 556 & 3.90 & 0.36 & 1.05 & 5.00 & 0.72 & $5.64 \mathrm{E}-02$ & 0.55 & 7.4 \\
\hline $06 / 09 / 2001$ & 14.4 & 7.45 & 557 & 3.97 & 0.34 & 1.04 & 5.09 & 0.73 & $4.97 \mathrm{E}-02$ & 0.49 & 5.0 \\
\hline $29 / 12 / 2001$ & 13.6 & 7.44 & 512 & 3.85 & 0.22 & 0.99 & 4.96 & 0.84 & $4.74 \mathrm{E}-02$ & 0.43 & 7.8 \\
\hline $18 / 02 / 2002$ & n.a & n.a & n.a & n.a & n.a & n.a & n.a & n.a & n.a & n.a & 9.3 \\
\hline $28 / 06 / 2002$ & n.a & n.a & n.a & n.a & n.a & n.a & n.a & n.a & n.a & n.a & 8.0 \\
\hline $28 / 12 / 2002$ & n.a & n.a & n.a & n.a & n.a & n.a & n.a & n.a & n.a & n.a & 7.6 \\
\hline
\end{tabular}

note: Cond $=$ electrical conductivity in $\mu \mathrm{S} \mathrm{cm}^{-1}$; ions and cations concentration in meq $1^{-1}$; iron in $\mu \mathrm{g}^{-1}$. 
Table 6. Chemical composition of the S. Pietro 2 well water.

\begin{tabular}{|c|c|c|c|c|c|c|c|c|c|c|c|}
\hline Date & $T^{\circ} \mathrm{C}$ & $p H$ & Cond & $\mathrm{HCO}_{3}^{-}$ & $\mathrm{Cr}$ & $\mathrm{SO}_{4}^{2-}$ & $\mathrm{Ca}^{2+}$ & $\mathrm{Mg}^{2+}$ & $\boldsymbol{K}^{+}$ & $\mathrm{Na}^{+}$ & $\mathrm{Fe}$ \\
\hline $10 / 05 / 1995$ & 13.8 & 7.62 & 589 & 3.90 & 0.31 & 0.80 & 4.48 & 0.56 & $5.38 \mathrm{E}-02$ & 0.46 & 8.9 \\
\hline $14 / 12 / 1995$ & 12.8 & 7.52 & 595 & 4.13 & 0.32 & 0.81 & 5.01 & 0.45 & $1.08 \mathrm{E}-01$ & 0.46 & 4.6 \\
\hline $17 / 06 / 1996$ & 14.0 & 7.69 & 585 & 4.02 & 0.29 & 0.84 & 4.89 & 0.59 & $5.38 \mathrm{E}-02$ & 0.41 & 3.9 \\
\hline $15 / 10 / 1996$ & 13.5 & 7.58 & 591 & 3.77 & 0.32 & 0.84 & 4.39 & 0.77 & $4.00 \mathrm{E}-02$ & 0.37 & 4.1 \\
\hline $18 / 11 / 1997$ & 13.0 & 7.34 & 589 & 3.80 & 0.35 & 0.84 & 4.44 & 0.69 & $3.54 E-02$ & 0.46 & 15.1 \\
\hline $23 / 03 / 1998$ & 13.6 & 7.70 & 507 & 3.70 & 0.29 & 0.74 & 4.32 & 0.73 & $3.61 \mathrm{E}-02$ & 0.43 & 9.6 \\
\hline $13 / 05 / 1998$ & 14.0 & 7.69 & 500 & 3.77 & 0.29 & 0.77 & 4.39 & 0.67 & $3.56 \mathrm{E}-02$ & 0.43 & 9.3 \\
\hline 18/05/1998 & 13.4 & 7.84 & 500 & 3.89 & 0.29 & 0.78 & 4.37 & 0.68 & $3.59 \mathrm{E}-02$ & 0.46 & 9.3 \\
\hline $15 / 12 / 1998$ & 13.0 & 7.33 & 500 & 3.84 & 0.31 & 0.79 & 4.46 & 0.71 & $3.59 \mathrm{E}-02$ & 0.47 & 9.4 \\
\hline 29/03/1999 & 13.4 & 7.35 & 509 & 3.82 & 0.29 & 0.76 & 4.47 & 0.68 & $3.72 \mathrm{E}-02$ & 0.44 & 9.4 \\
\hline $24 / 06 / 1999$ & 14.2 & 7.42 & 483 & 3.74 & 0.27 & 0.80 & 4.37 & 0.67 & $3.85 \mathrm{E}-02$ & 0.45 & 8.9 \\
\hline $28 / 09 / 1999$ & 13.5 & 7.40 & 496 & 3.75 & 0.28 & 0.80 & 4.38 & 0.67 & $3.41 \mathrm{E}-02$ & 0.44 & 8.4 \\
\hline $11 / 12 / 1999$ & 13.2 & 7.52 & 502 & 3.56 & 0.29 & 0.83 & 4.15 & 0.59 & $2.82 \mathrm{E}-02$ & 0.47 & 8.7 \\
\hline $24 / 03 / 2000$ & 13.4 & 7.43 & 439 & 4.23 & 0.25 & 0.75 & 4.93 & 0.65 & $4.05 \mathrm{E}-02$ & 0.44 & 5.1 \\
\hline $24 / 06 / 2000$ & 13.8 & 7.52 & 488 & 4.26 & 0.27 & 0.82 & 4.97 & 0.69 & $4.10 \mathrm{E}-02$ & 0.43 & 5.7 \\
\hline $25 / 09 / 2000$ & 14.3 & 7.42 & 463 & 3.93 & 0.27 & 0.72 & 4.60 & 0.67 & $4.79 \mathrm{E}-02$ & 0.43 & 5.4 \\
\hline $09 / 12 / 2000$ & 12.2 & 7.99 & 461 & 3.97 & 0.28 & 0.72 & 4.63 & 0.69 & $4.87 \mathrm{E}-02$ & 0.50 & 5.3 \\
\hline $24 / 03 / 2001$ & 13.8 & 7.51 & 516 & 4.00 & 0.30 & 0.77 & 4.71 & 0.68 & $4.67 \mathrm{E}-02$ & 0.47 & 6.1 \\
\hline $21 / 05 / 2001$ & 14.0 & 7.75 & 508 & 4.20 & 0.33 & 0.85 & 4.88 & 0.67 & $4.61 \mathrm{E}-02$ & 0.42 & 6.2 \\
\hline $06 / 09 / 2001$ & 14.3 & 7.50 & 504 & 4.02 & 0.33 & 0.84 & 4.76 & 0.72 & $4.56 \mathrm{E}-02$ & 0.43 & 5.6 \\
\hline $29 / 12 / 2001$ & 13.4 & 7.41 & 462 & 3.77 & 0.21 & 0.75 & 4.94 & 0.75 & $3.33 \mathrm{E}-02$ & 0.43 & 5.3 \\
\hline $18 / 02 / 2002$ & n.át & n.a & n.at & n.a & n.a & n.a & n.a & n.a & n.a & n.a & 5.3 \\
\hline $28 / 06 / 2002$ & n.a & n.a & n.a & n.a & n.a & n.a & n.a & n.a & n.a & n.a & 4.2 \\
\hline $28 / 12 / 2002$ & n.a & n.a & n.a & n.a & n.a & n.a & n.a & n.a & n.a & n.a & 2.7 \\
\hline
\end{tabular}

note: Cond $=$ electrical conductivity in $\mu \mathrm{S} \mathrm{cm}^{-1}$; ions and cations concentration in meq $1^{-1}$; iron in $\mu \mathrm{g}^{-1}$. 
Table 7. Temperature, $\mathrm{pH}$ and electrical conductivity data for the Cassignano spring. Cond $=$ electrical Conductivity in $\mu \mathrm{S} \mathrm{cm}^{-1}$.

\begin{tabular}{cccc}
\hline Date & $\boldsymbol{T}^{\mathrm{C}} \boldsymbol{C}$ & $\boldsymbol{p} \boldsymbol{H}$ & Cond \\
\hline $16 / 10 / 1992$ & n.a & 7.52 & 324 \\
$10 / 02 / 1993$ & n.a & 7.69 & 271 \\
$06 / 02 / 1995$ & n.a & 7.56 & 286 \\
$27 / 09 / 1997$ & n.a & 7.55 & 429 \\
$03 / 10 / 1997$ & n.a & 7.33 & 492 \\
$14 / 10 / 1997$ & 13.4 & 7.58 & 431 \\
$16 / 11 / 1997$ & n.a & 7.37 & 418 \\
$06 / 06 / 1998$ & 12.3 & 7.38 & 342 \\
$29 / 03 / 1999$ & 8.7 & 7.63 & 324 \\
$24 / 06 / 1999$ & 14.7 & 7.61 & 318 \\
$28 / 09 / 1999$ & 13.4 & 7.62 & 336 \\
$11 / 12 / 1999$ & 9.7 & 8.11 & 331 \\
$24 / 06 / 2000$ & 14.6 & 7.72 & 300 \\
$25 / 09 / 2000$ & 13.0 & 7.67 & 310 \\
$09 / 12 / 2000$ & 11.6 & 7.68 & 300 \\
$24 / 03 / 2001$ & 12.1 & 7.65 & 313 \\
$21 / 05 / 2001$ & 12.1 & 7.66 & 315 \\
$12 / 09 / 2001$ & 13.9 & 7.64 & 337 \\
$19 / 02 / 2002$ & 8.5 & 7.84 & 301 \\
$28 / 06 / 2002$ & 16.4 & 7.80 & 300 \\
$28 / 12 / 2002$ & 9.9 & 7.81 & 335 \\
\hline
\end{tabular}

Table 8. Temperature, $\mathrm{pH}$ and electrical conductivity data for the Mattinate spring. Cond $=$ electrical conductivity in $\mu \mathrm{S} \mathrm{cm}^{-1}$.

\begin{tabular}{cccc}
\hline Date & $\boldsymbol{T}^{\circ} \boldsymbol{C}$ & $\boldsymbol{p H}$ & $\boldsymbol{C o n d}$ \\
\hline $10 / 02 / 1993$ & n.a & 7.74 & 296 \\
$23 / 09 / 1993$ & n.a & 7.70 & 348 \\
$06 / 02 / 1995$ & n.a & 7.77 & 339 \\
$27 / 09 / 1997$ & n.a & 7.66 & 435 \\
$30 / 09 / 1997$ & n.a & 7.62 & 475 \\
$03 / 10 / 1997$ & n.a & 7.27 & 470 \\
$06 / 06 / 1998$ & 10.0 & 7.58 & 293 \\
$29 / 03 / 1999$ & 9.7 & 7.62 & 339 \\
$24 / 06 / 1999$ & 12.2 & 7.63 & 333 \\
$28 / 09 / 1999$ & 14.2 & 7.58 & 337 \\
$11 / 12 / 1999$ & 9.4 & 7.96 & 369 \\
$24 / 06 / 2000$ & 11.7 & 7.58 & 328 \\
$26 / 09 / 2000$ & 13.2 & 7.62 & 320 \\
$09 / 12 / 2000$ & 11.3 & 7.51 & 330 \\
$24 / 03 / 2001$ & 9.9 & 7.64 & 359 \\
$21 / 05 / 2001$ & 10.1 & 7.62 & 356 \\
$12 / 09 / 2001$ & 11.8 & 7.63 & 347 \\
$19 / 02 / 2002$ & 7.8 & 7.93 & 306 \\
$28 / 06 / 2002$ & 12.1 & 7.68 & 300 \\
$28 / 12 / 2002$ & 9.2 & 7.63 & 351 \\
\hline
\end{tabular}

intermediate composition (i.e., mixing between a sulphate and a chlorine-rich water with carbonate waters; Stifone spring). The composition of the cold waters was modified during the seismic crisis due to the addition of sulphate-rich waters. The proposed results, which also show that cold waters may suffer from vast modifications on account of seismic activity, provide information complementary to that supplied by the thermal waters.

\section{TEMPORAL VARIATIONS}

The temporal variations are shown in Figs. 4 - 7 (respectively for electrical conductivity, $\mathrm{pH}, \mathrm{Fe}$ and $\mathrm{SO}_{4}{ }^{2-}$ contents), where the geochemical parameters are plotted versus time. The geochemical data are superimposed on the magnitude of those shocks having $\mathrm{M}>3.5$ that occurred in a circular area with a radius of $50 \mathrm{~km}$ around the epicentral (Colfiorito) area of the 1997 - 98 seismic swarm.

As already pointed out, at the beginning of the seismic crisis modifications in the geochemical parameters were recorded in the thermal waters of the Region (Favara et al. 2001, Caracausi 


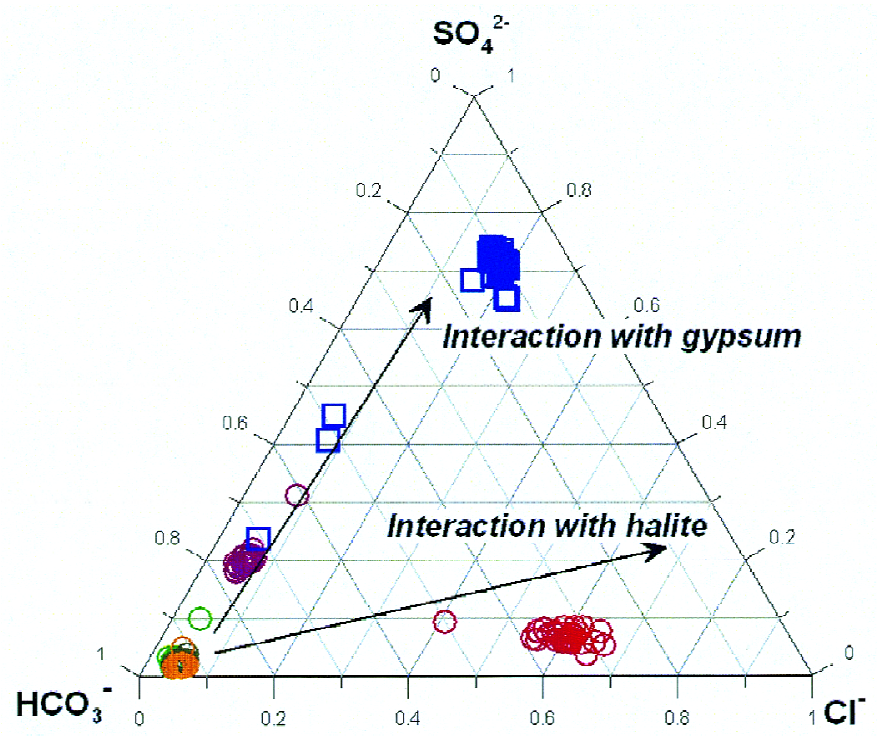

Fig. 3. Triangular diagram $\mathrm{SO}_{4}{ }^{2-}-\mathrm{HCO}_{3}{ }^{-}-\mathrm{Cl}^{-}$. The graph shows the different water groups and highlights the difference between cold (empty circular marks) and thermal waters (squared marks). The cold waters display a clear mixing with a component circulating in the deep selenitic rocks in relation to 1997 - 1998 seismic period. Symbols same as in Fig. 2.

because of its relatively high solubility in water, and produces carbonic acid. Increased concentration of $\mathrm{HCO}_{3}{ }^{-}$were recorded in several cold waters (e.g., Colfiorito well, Capodacqua; see Tables 1 to 8 ). Besides the changes in the bicarbonate contents, the chemical analyses highlight an increase in $\mathrm{SO}_{4}{ }^{2-}$. It is worthwhile noting that the sulphate ions come from the deep selenitic rocks that normally host the thermal waters, and thus show the presence of a water component having a different origin than limestones and alluvional terrains where the cold waters circulate. This information, as well as the increased electrical conductivity and the decreased $\mathrm{pH}$ values, lead us to conclude that two main phenomenon modified the geochemical features of the cold waters: an increased $\mathrm{CO}_{2}$ input and mixing with a water component, equilibrated with gypsum, that is normally confined to deeper levels and does not interact with the shallow circulating waters. The mixed water component is the same thermal component that normally outflows from the Bagni di Triponzo springs (see Figs. 2, 3).

Another anomaly we detected was the increase in iron concentration. The iron content started to increase well before the beginning of the seismic crisis (i.e., three months) and provides us with clues of pre-seismic modifications. Iron concentration was back to background values about one year later. This modification of the iron content is significant as it may have been caused either by the addition of deep-circulating waters to the shallow, cold waters normally exploited for drinking purposes, or by the remobilization of iron from iron-In coincidence with the 26 March, 1998 

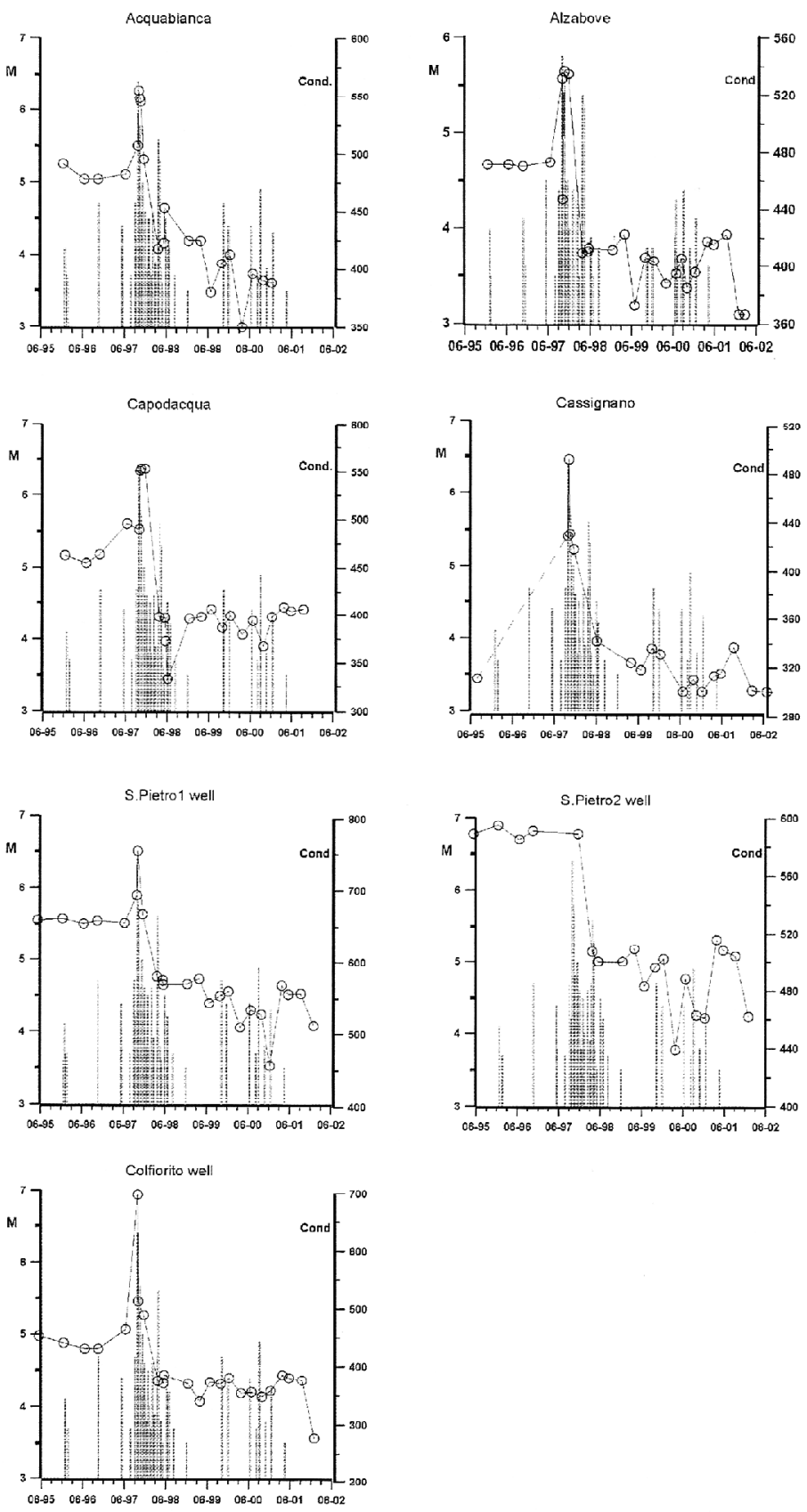

Fig. 4. Temporal variations of electrical conductivity (Cond) recorded in cold well and spring waters. The values are expressed in $\mu \mathrm{S} \mathrm{cm}^{-1}$. The first datum for Cassignano refers to the average value between 1992 and 1995. 

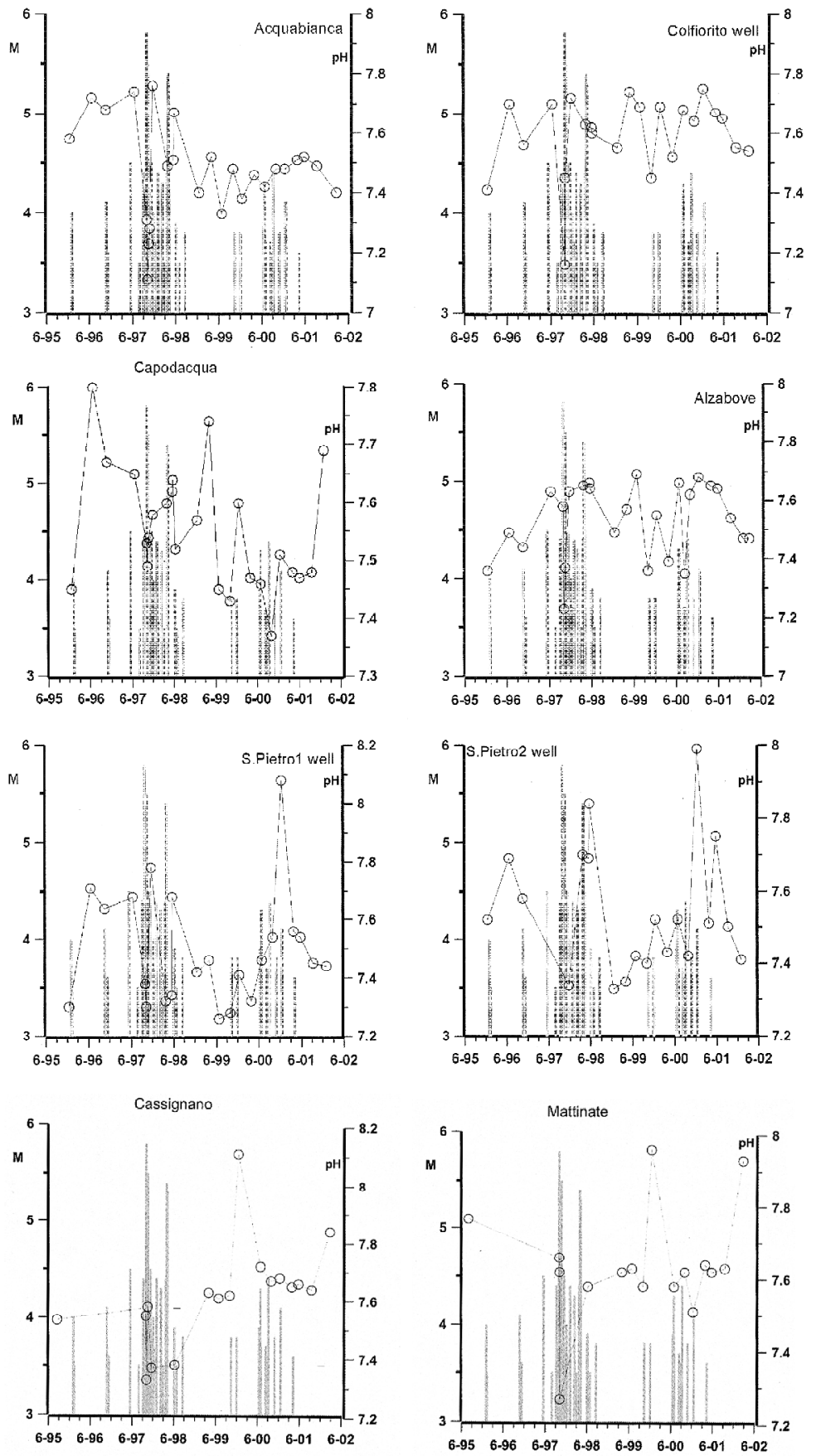

Fig. 5. Temporal variations of $\mathrm{pH}$ of the cold waters from wells and springs. 

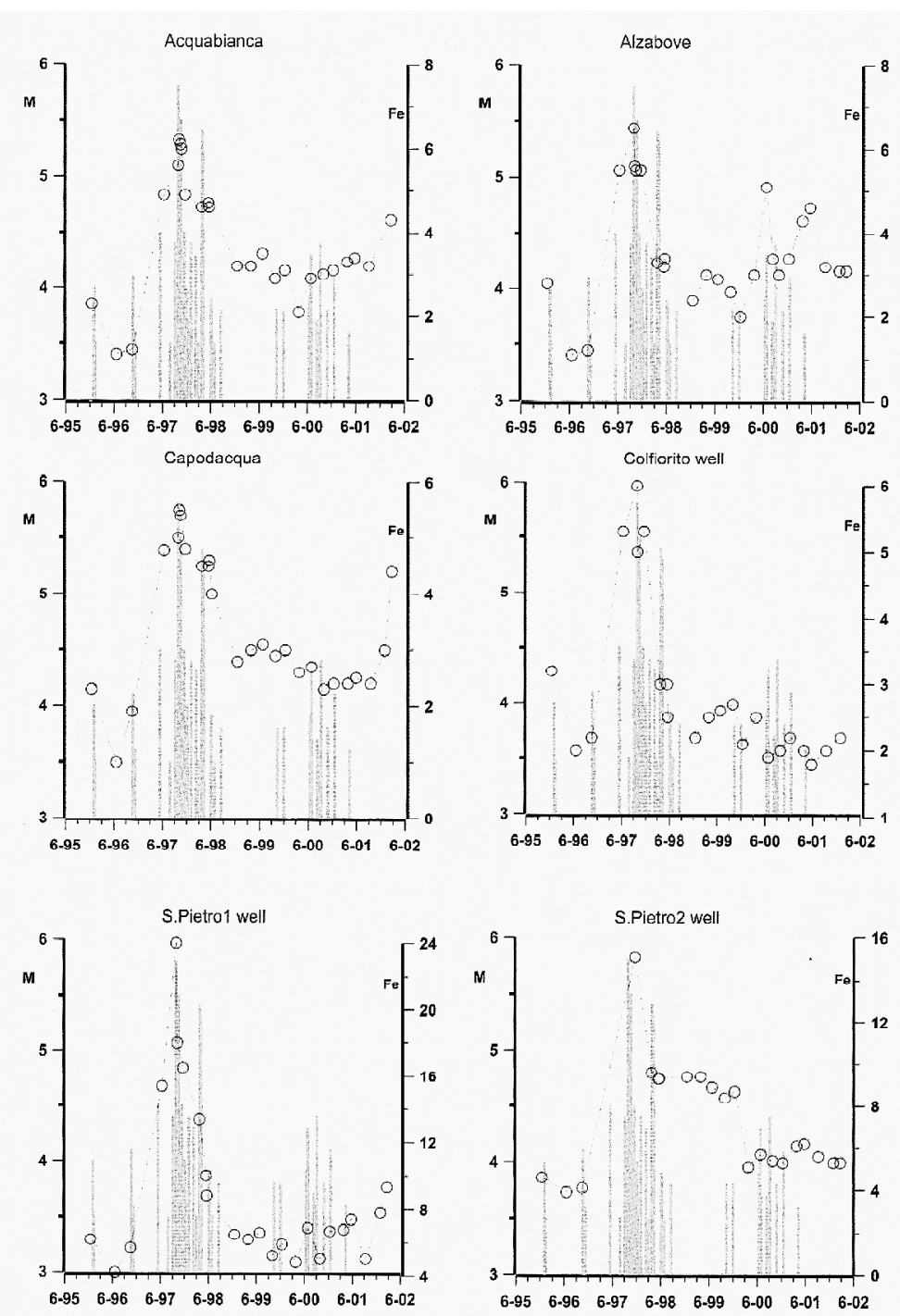

Fig. 6. Temporal variations of the iron content. Concentration units in $\mu \mathrm{g}^{-1}$.

rich mineralogical phases that are a common feature in the carbonate rocks of Umbria. Iron mobilization is mainly a consequence of variations in the Redox potential (Garrels 1960). Redox conditions can be modified either as a consequence of mixing with deep-circulating waters or because of interactions with a reducing deep-originated gas-phase. It should be noted that the analysis of the dissolved gases in the thermal waters highlighted the presence of a $\mathrm{CO}_{2}$-dominated gas component mixed with the normal gaseous components of an air-saturated water (ASW) (Italiano et al. 2004). 

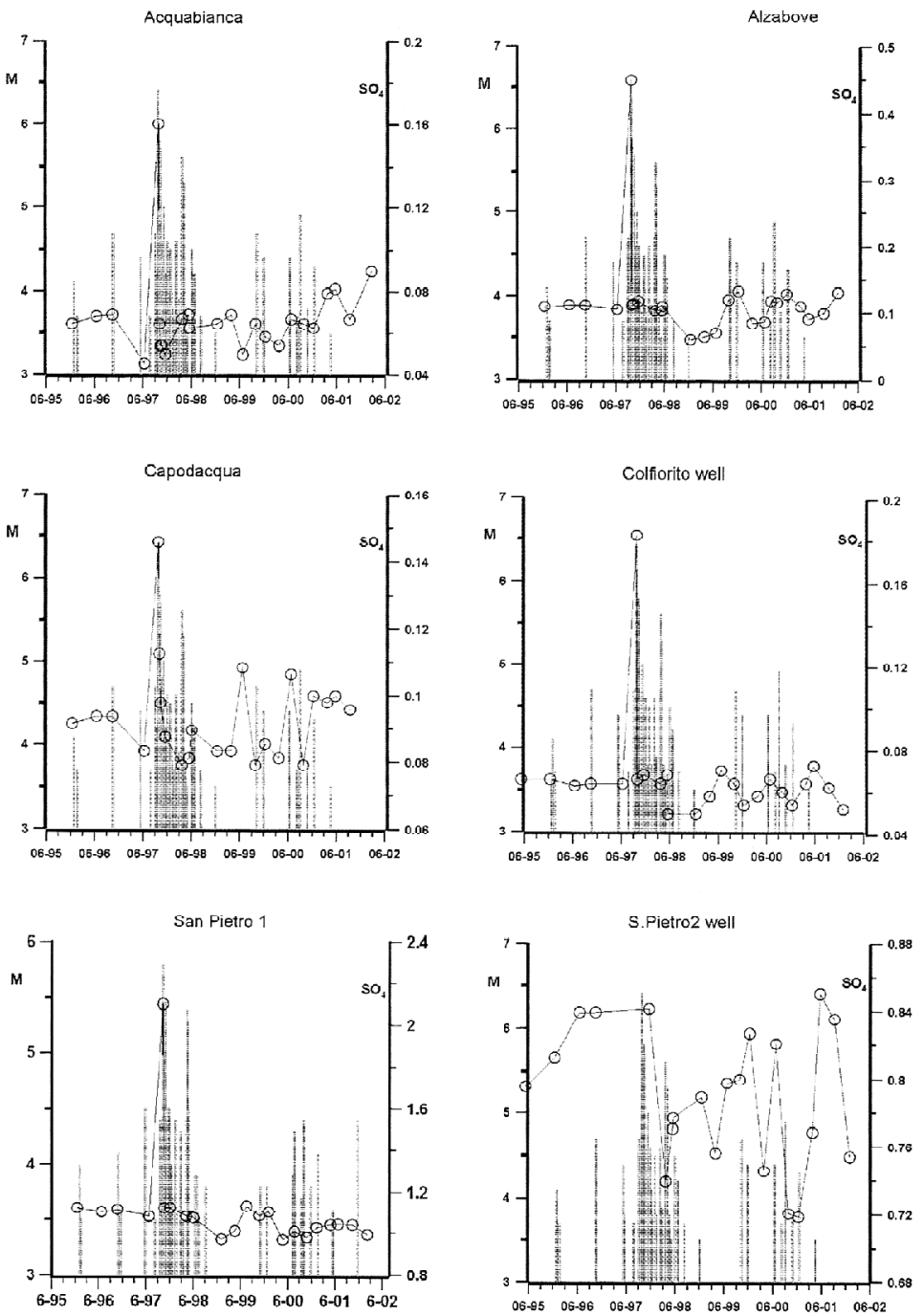

Fig. 7. Temporal variations of $\mathrm{SO}_{4}$ concentrations. The values are expressed in meq $1^{-1}$.

seismic shock (whose epicentral area was located north of Colfiorito) the water from both the Acquabianca spring and the S. Pietro 1 well underwent another drop in $\mathrm{pH}$ values (Fig. 5), in contrast to the increase in the values recorded at all the cold and thermal waters. Such divergent behaviour, observed both in coincidence with the 1997 and 1998 seismic events and among the various sampling sites, might be related to the different permeability variations induced by the faulting activity, which was clearly extensive during the 1997 event (Basili and Meghraoui 2001) but compressive in March, 1998 (Morelli et al. 2000). 


\section{CONCLUSIONS}

The observations made during the geochemical monitoring activity in Umbria allowed us to detect interesting behavior on the part of the circulating fluids during the 1997 - 98 seismic crisis. The ample literature on the subject [see Thomas (1988) and cited references; Toutain and Baubron (1999) and literature therein] and more recently Ramirez-Guzman et al. (2005), Song et al. (2005) shows that seismogenic-induced geochemical modifications occur in anomalous fluids (i.e., thermal waters, acidic gases, etc.) circulating in seismic-prone areas. This time we have shown that fluids (cold waters) that are normally exploited for drinking purposes can also suffer from geochemical modifications induced by seismicity, or, more realistically, by the development of the seismogenic process itself.

The evidence that the widest geochemical anomalies occurred only at the beginning of the seismic crisis, when tensile faulting was responsible for the M 5.9 and 5.5 shocks, demonstrates that the fault rupture increased vertical permeability thereby allowing the shallow waters to be contaminated by deep-originated components. The $\mathrm{SO}_{4}{ }^{2-}$ concentrations of the thermal waters highlight significant variations during both the 1997 and 1998 earthquake sequences: a pre-seismic increase and a sharp decrease that coincided with the beginning of the two seismic swarms.

The investigated wells and springs are located along the seismogenetic normal faults that mark the eastern sector of Umbria, where the strongest earthquakes in the region have occurred (Collettini and Barchi 2004). Our results show that despite the fact that there are no significant field evidences of tectonic discontinuities, and that no faulting activity was recorded during the seismicity (De Martini et al. 2003), the geochemical variations recorded in those cold waters apparently not located along fault-lines (i.e., see the $\mathrm{pH}$ drop in the water of Mattinate spring, Fig. 5) show that the presence of fluids can often be related to faults while temporal variations can be a clue to fault movements.

Although the concentrations of some trace elements, dangerous for human health, were below the threshold values for human safety (i.e., $50 \mu \mathrm{g} 1^{-1}$ for $\mathrm{Fe}$ ), the possibility that shallow groundwaters could be contaminated during seismic activity by deep-originated fluids has to be taken into consideration when dealing with seismic hazard evaluations.

Another important aspect is related to environmental considerations. Since it is evident that groundwaters can also be contaminated by natural processes, long-term geochemical monitoring would provide useful information both for scientific and practical purposes.

\section{REFERENCES}

Amato, A., R. Azzara, C. Chiarabba, G. B. Cimini, M. Cocco, M. Di Bona, L. Margheriti, S. Mazza, F. Mele, G. Selvaggi, A. Basili, E. Boschi, F. Courboulex, A. Dechamps, S. Gaffet, G. Bittarelli, L. Chiaraluce, D. Piccinini, and M. Ripepe, 1998: The 1997 UmbriaMarche, Italy, earthquake sequence: a first look at the main shocks and aftershocks. Geophs. Res. Lett., 25, 2861-2864. 
Barchi, M. R., A. De Feyter, B. Magnani, G. Minelli, G. Pialli, and B. M. Sotera, 1998: The structural style of the Umbria-Marche fold and thrust belt.Mem. Soc. Geol. It., 52, 557-578.

Barchi, M. R., 2002: Lithological and structural controls on the seismogenesis of the Umbria region: observations from seismic reflection profiles. Bull. Soc. Geol. It., 1, 855-864.

Basili, R., and M. Meghraoui, 2001: Coseismic and postseismic displacements related with the 1997 earthquake sequence in Umbria-Marche (central Italy). Geophys. Res. Lett., 28, 2695-2698.

Bazzoffia, A., A. Caracausi, P. Innocenzi, F. Italiano, and G. Martinelli, 2002: Variazioni geochimiche in acque sotterranee fredde dell'Umbria connesse con la sequenza sismica del 1997-1998. Acque Sotterranee, 78, 23-33. (in Italian)

Boncio, P., F. Brozzetti, F. Ponziani, M. Barchi, G. La Vecchia, and G. Pialli, 1998: Seismicity and extensional tectonics in the Northern Umbria-Marche Apennines. Mem. Soc. Geol. It., 52, 539-555.

Castello B., G. Selvaggi, C. Chiarabba, and A. Amato, 2005: CSI Catalogo della sismicità italiana 1981-2002, versione 1.0. INGV-CNT, Roma. http://www.ingv.it/CSI/

Caracausi, A., F. Italiano, G. Martinelli, A. Paonita, and A. Rizzo, 2005: Long-term geochemical monitoring and extensive/compressive phenomena: case study of the Umbria region (central Apennines, Italy). An. Geophys., 48, 43-53.

Chiodini, G., S. Giaquinto, and A. R. Zanzari, 1982: Relazione tra il chimismo delle sorgenti Umbre e le caratteristiche litologiche degli acquiferi. In: Energia Geotermica, Prospettive Aperte dalle ricerche geotermiche del CNR, Roma Dicembre 1982, 30-36. (in Italian)

Collettini, C., and M. R. Barchi, 2002: A low-angle normal fault in the Umbria region (Central Italy): a mechanical model for the related microseismicity. Tectonophys., 359, 97-115.

Collettini, C., and M. R. Barchi, 2004: A comparison of structural data and seismic images for low-angle normal faults in the Northern Apennines (Central Italy): constraints on activity. In: Alsop, G. I., R. E. Holdsworth, K. J. W. McCaffrey, and M. Hand (Eds.), Flow Processes in Faults and Shear Zones. Geol. Soc., London, Spec. Publ., 224, 95-112.

De Martini, P. M., N. A. Pino, G. Valensise, and S. Mazza, 2003: Geodetic and seismologic evidence for slip variability along a blind normal fault in the Umbria-Marche 19971998 earthquakes (central Italy). Geophys. J. Int., 155, 819-829.

Favara, R., F. Italiano, and G. Martinelli, 2001: Earthquake-induced chemical changes in thermal waters of Umbria region during the 1997-1998 seismic swarm. Terra Nova, 13, 227-233.

Garrels, R. M., 1960: Mineral Equilibria. Harper and Brothers Publishers, New York, 254 pp.

Giaquinto, S., G. Marchetti, A. Martinelli, and E. Martini, 1991: Le acque sotterranee in Umbria, Principali studi condotti dalla Regione Umbria. Pubblicazione $n .413$ del GNDCI-CNR, 49-64, Casa Editrice Perugia Pitagora.

Heinicke, J., F. Italiano, V. Lapenna, G. Martinelli, and P. M. Nuccio, 2000: Coseismic geochemical variations in some gas emissions of Umbria region (Central Italy).Phys. Chem. Earth, 25, 289-293.

Italiano, F., G. Martinelli, and P. M. Nuccio, 2001: Anomalies of mantle-derived helium during the 1997-1998 seismic swarm of Umbria-Marche, Italy. Geophys. Res. Lett., 28, 839-842. 
Italiano F., G. Martinelli, and A. Rizzo, 2004: Seismogenic-induced variations in the dissolved gases of the thermal waters of the Umbria region (Central Apennines, Italy) during and after the 1997-98 seismic swarm. Geochem. Geophys. Geosyst., 5, 11, doi: 10.1029/2004GC000720.

Langelier, W. F., and H. F. Ludwing, 1942: Graphical methods for indicating the mineral character of natural waters. J. Am. Water Works Assoc., 34, 335.

La Vecchia, G., F. Brozzetti, M. R. Barchi, M. Menichetti, and J. V. A. Keller, 1994: Seismotectonics zoning in east-central Italy deduced from an analysis of the Neogene to present deformations and related stress fields. Geol. Soc. Am. Bull., 106, 1107-1120.

Montone, P., A. Amato, and S. Pondrelli, 1999: Active stress map of Italy.J. Geophys. Res., 40, 25595-25610.

Morelli, A., G. Ekstrom, and M. Oliveri, 2000: Source properties of the 1997-1998 Central Italy earthquake sequence from inversion of long-period and broad-band seismograms. J. Seismol., 4, 365-375.

Quattrocchi, F., R. Pik, L. Pizzino, M. Guerra, P. Scarlato, M. Angeolone, M. Barbieri, A. Conti, B. Marty, E. Sacchi, G. M. Zuppi, and S. Lombardi, 2000: Geochemical Changes at the Bagni di Triponzo thermal spring during the Umbria-Marche 1997-1998 seismic sequenze. J. Seismol., 4, 567-587.

Ramirez-Guzman, A., Y. A. Taran, R. Bernard, E. Cienfuegos, and P. Morales, 2005: Variations in the $\mathrm{Cl}, \mathrm{SO}_{4}, \delta \mathrm{D}$ and $\delta^{18} \mathrm{O}$ in water from thermal springs near Acapulco, Guerrero, Mexico, related to seismic activity. Terr. Atmos. Ocean. Sci., 16, 731-743.

Song, S. R., Y. L. Chen, C. M. Liu, W. Y. Ku, H.F. Chen, Y. J. Liu, L. W. Kuo, T. F. Yang, C. H. Chen, T. K. Liu, and M. Lee, 2005: Hydrochemical changes of spring waters in Taiwan: implications for evaluating sites for earthquake precursory monitoring. Terr. Atmos. Ocean. Sci., 16, 745-762.

Thomas, D., 1988: Geochemical precursors to seismic activity. Pure Appl. Geophys., 126, 241-265.

Toutain, J. P., and J. C. Baubron, 1999: Gas geochemistry and seismotectonics: a review. Tectonophys., 304, 1-24.

Zanzari, A. R., A. Martinelli, R. Cioni, M. Guidi, B. Raco, A. Scozzari, F. Quattrocchi, G. Galli, and C. Mancini, 2001: Discrete and continuous monitoring of groundwaters in the seismic area of the Umbria Region (Italy). WRI 2001, Cidu (Ed.), 1, 119-122. 